Tohoku Math. J.

54 (2002), 105-120

\title{
A FREE BOUNDARY VALUE PROBLEM OF EULER SYSTEM ARISING IN SUPERSONIC FLOW PAST A CURVED CONE
}

\author{
SHUXING CHEN
}

(Received April 5, 2000, revised July 23, 2001)

\begin{abstract}
We study a free boundary value problem of the Euler system arising in the inviscid steady supersonic flow past a symmetric curved cone. The existence and stability of piesewise smooth weak entropy solutions was established, provided the cone is a small perturbation of its tangential cone with a vertex angle less than a given value determined by the parameters of the coming flow. Since the change of the entropy of the flow is also considered, the result in this paper gives a more precise description than previous ones on such problems.
\end{abstract}

1. Introduction. This paper is devoted to the study of a free boundary value problem of the Euler system, which arises in the inviscid steady supersonic flow past a symmetrically curved cone. It is well-known that when a supersonic flow passes a pointed body, there appears a shock front ahead of the body. If the body has a blunt head, the shock in front of it is detached. Otherwise, if the body has sharp head, the shock can be attached on it. Such phenomena have been verified by physical experiments and numerical computation. However, the problem is solved only in some special cases. It is indicated in [5] that if the body is a circular cone with a vertex angle less than a critical value, determined by the parameters of the coming flow, then the solution can be determined by solving a boundary value problem of an ordinary differential equation. In this case a shock front will be generated and attached at the tip of the cone. The shock also forms a circular cone with the same axis as the surface of the solid body. Under the additional assumption of the weakness of the shock, the flow behind the shock can be described by a potential equation. Under such a framework we proved in [4] that the flow with the attached shock is stable, if the body becomes a perturbed curved cone. This means that, we still have the existence of solutions with attached shock near the vertex of the perturbed conical body. However, the potential equation is only a description of an isentropic and irrotational flow. When the conical body is not an exact circular cone, the attached shock is also not a circular cone. It turns out that the entropy in the flow is not constant in fact. Therefore, it is natural to work with the full Euler system, which is more precise description of the inviscid steady flow. Certainly, the problem becomes more difficult, because generally the boundary value problem for differential system is more complicated than the corresponding problem for a second order equation.

2000 Mathematics Subject Classification. Primary 35L60; Secondary 35L67, 35L45.

The work is partially supported by NNSF of China, the Doctoral Foundation of NEC, the Key Grant of NMST of China and the Hatori Foundation. The author also greatly appreciates the hospitality of IMS of Chinese University of Hong Kong, where he finally completed this paper. 
In this paper, we are going to deal with the situation for a uniform supersonic flow past a symmetricaly curved cone in the framework of the Euler system. As in $[3,4]$, we call the tangential circular cone as the background cone, and the solution to the coming supersonic flow past the background cone as the background solution. Assume that the background cone is tangential to the curved cone in higher order. Then we confirm in this paper that there exists a local solution with a conical curved shock attached at the tip of the solid body, provided that the vertex angle of the tangential cone is less than a critical value, determined by the parameters of the coming flow. Besides, the flow field and the location of the shock are stable with respect to the perturbation of the surface of the solid body. The solution obtained here is piesewise smooth, i.e., the equation and the boundary conditions are satisfied in a classical sense. We also mention that the author of [12] also discuss a similar problem in the BV space by using the Glimm scheme.

Let us briefly describe the approach to solve our problem here. It is convenient to discuss our problem in the cylindrical coordinates. In the domain where a solution of the Euler system is smooth, the system can be reduced to a symmetric hyperbolic one. On the surface of the body the flow satisfies the rigid wall condition, and on the shock front the well-known Rankine-Hugoniot conditions hold. The solution of this nonlinear problem will be obtained through linearization and Newton's iteration. Hence as a necessary step we need to construct a good first approximation, which describes the solution with high accuracy near the vertex. Then we gradually improve the degree of approximation. Usually, the approximate solution can be constructed by Taylor expansion with solving a series of algebraic equations. However, because of the singularity of the domain at the vertex of the cone, we have to reduce the construction of the approximate solution to solving a series of boundary value problems of a differential system. The first boundary value problem is a nonlinear one, and it is nothing but the problem in a symmetric case. All other problems are linear one, and the corresponding systems have the same principal part as the first one. Under our assumptions on the higher order tangency, we only need to consider the solvability of the system with large index. Since these systems with large index are symmetric positive one, and the linearized boundary conditions both on the surface body and on the shock are admissible according to the terminology in the theory of symmetric positive systems (see $[6,8]$ ). Therefore, all these linear problems can be solved perfectly. By using these solutions, we obtain an approximate solution of the original nonlinear problem with error $O\left(z^{N}\right)$. Afterwards, we establish the estimates for the linearization of the nonlinear problem, and then we are led to to the expected solution of the nonlinear problem by using Newton's iteration.

The paper is arranged as follows. In Section 2, we formulate the problem and introduce a singular conic coordinates, which amounts to the angle in the polar coordinate system. Then we recall some basic results on solutions of the symmetric conic shocks and state our main theorem of this paper precisely. In Section 3, we deduce a set of boundary value problem by using finite expansion, and then construct an approximate solution after solving these problems. In Section 4, we first rewrite the nonlinear problem and establish the energy estimates for the linearized problem. Then in Section 5, we use the approximate solution established in 
Section 3 as the first term in the linear iteration and obtain the local existence for the original nonlinear problem. Meanwhile, we will also indicate that the solution is stable with respect to the perturbation of the surface of the body.

2. Formulation of problem. The system for the axi-symmetric inviscid steady flow in the cylindrical coordinates takes the form

$$
\frac{\partial}{\partial z}\left(\begin{array}{c}
\rho u_{z} \\
p+\rho u_{z}^{2} \\
\rho u_{z} u_{r} \\
u_{z}(E+p)
\end{array}\right)+\frac{\partial}{\partial r}\left(\begin{array}{c}
\rho u_{r} \\
\rho u_{z} u_{r} \\
p+\rho u_{r}^{2} \\
u_{r}(E+p)
\end{array}\right)+\frac{1}{r}\left(\begin{array}{c}
\rho u_{r} \\
\rho u_{z} u_{r} \\
\rho u_{r}^{2} \\
u_{r}(E+p)
\end{array}\right)=0
$$

where $\left(u_{z}, u_{r}\right)$ stands for the velocity, $q^{2}=u_{r}^{2}+u_{z}^{2}, \rho$ is the density, $p$ is the pressure, $E=\rho\left(e+q^{2} / 2\right)$ is the total energy with $e$ being the internal energy. Denoting $\left(u_{z}, u_{r}\right)$ by $(u, v)$, the system $(2.1)$ can also be written as

$$
\begin{aligned}
& \frac{\partial}{\partial z}(\rho u)+\frac{\partial}{\partial r}(\rho v)+\frac{1}{r} \rho v=0 \\
& u \frac{\partial u}{\partial z}+v \frac{\partial u}{\partial r}+\frac{1}{\rho} \frac{\partial p}{\partial z}=0 \\
& u \frac{\partial v}{\partial z}+v \frac{\partial v}{\partial r}+\frac{1}{\rho} \frac{\partial p}{\partial r}=0 \\
& u \frac{\partial}{\partial z}\left(e+\frac{p}{\rho}+\frac{1}{2} q^{2}\right)+v \frac{\partial}{\partial r}\left(e+\frac{p}{\rho}+\frac{1}{2} q^{2}\right)=0
\end{aligned}
$$

in the domain where the solution is smooth.

Consider a uniform flow coming from infinity and attacking a symmetrically curved cone with its equation

$$
r=b(z)
$$

satisfying $b(0)=0$. It is well-known that the flow generates a shock, which is attached at the vertex of the curved cone, if $b^{\prime}(0)$ is small. Otherwise, the flow generates a detached shock, if $b^{\prime}(0)$ is large. On the boundary (2.3) the flow satisfies the boundary condition

$$
u_{r}-b^{\prime}(z) u_{z}=0 \text {. }
$$

In this paper we only consider the case when the shock in front of the body is attached. The equation of the shock is assumed as

$$
r=\sigma(z) .
$$

On the shock the well-known Rankine-Hugoniot condition holds:

$$
\sigma^{\prime}(z)\left[\begin{array}{c}
\rho u_{z} \\
p+\rho u_{z}^{2} \\
\rho u_{z} u_{r} \\
u_{z}\left(\rho e+p+\rho q^{2} / 2\right)
\end{array}\right]-\left[\begin{array}{c}
\rho u_{r} \\
\rho u_{z} u_{r} \\
p+\rho u_{r}^{2} \\
u_{r}\left(\rho e+p+\rho q^{2} / 2\right)
\end{array}\right]=0,
$$


where [.] means the jump of the corresponding quantities in the bracket. Besides, the entropy condition should be satisfied. The latter means that the normal component of the velosity behind the shock is subsonic, while the one ahead of the shock is supersonic.

The problem of supersonic flow past a symmetrically curved body is now formulated as the boundary value problem of the Euler system (2.1) in the domain $b(z) \leq r \leq \sigma(z)$. The solution is smooth in the domain, and should satisfy the boundary condition (2.4) on $r=b(z)$, and satisfy the condition (2.6) and the entropy condition on $r=\sigma(z)$. Here the function, describing the location of the shock front is also unknown, which should be determined together with the solution. In the sequel we shall prove the existence of a solution of the boundary value problem (2.1), (2.4), (2.6). The conclusion is

THEOREM 2.1. Let $\mathcal{C}$ be an axi-symmetrically curved cone with equation $r=b(z)$, where $b(z)$ is smooth and satisfies that $b(0)=0$, and $\partial^{k} b(0)=0$ for $2 \leq k \leq k_{0}$ with $k_{0}$ being a suitable integer. Assume that the coming flow is supersonic, $b_{1}=b^{\prime}(0)$ is less than the critical value determined by the parameters of the coming flow, and the background conical shock $r=s_{1} z$ corresponding to the cone $r=b_{1} z$ is a weak attached shock. Then for sufficiently small $z_{0}$ we can determine a function $\sigma(z)$ for $0<z<z_{0}$ and the solution of the system (2.1) in the domain $b(z)<r<\sigma(z), 0<z<z_{0}$, which satisfy the boundary conditions (2.4) on $r=b(z)$, the boundary condition (2.6) and the entropy condition on $r=\sigma(z)$. Meanwhile, the solution with such a structure is also unique and stable. Here the stability means that the small perturbation of $b(z)$ only causes the small perturbation of $\sigma(z)$ and the unknown function $U$.

REMARK 2.1. Since the coming flow is uniform, we have

$$
e+\frac{p}{\rho}+\frac{1}{2} q^{2}=\text { const. }
$$

ahead of the shock. Then, by using the last equation of (2.2) and (2.6), the relation (2.7) holds in the whole field. This is called Bernoulli's relation. $e+p / \rho$ is also called the enthalpy and usually is denoted by $i$. In the sequel (2.7) will be applied to replace the last equation of (2.1) or (2.2).

By using (2.7), the system (2.2) can be rewritten as a symmetric positive hyperbolic system

$$
\left(\begin{array}{ccc}
\rho u & 1 \\
& \rho u & \\
1 & & a^{-2} \rho^{-1} u
\end{array}\right) \frac{\partial}{\partial z}\left(\begin{array}{c}
u \\
v \\
p
\end{array}\right)
$$

$$
+\left(\begin{array}{ccc}
\rho v & & \\
& \rho v & 1 \\
& 1 & a^{-2} \rho^{-1} v
\end{array}\right) \frac{\partial}{\partial r}\left(\begin{array}{l}
u \\
v \\
p
\end{array}\right)+\frac{1}{r}\left(\begin{array}{l}
0 \\
0 \\
v
\end{array}\right)=0,
$$

which can also be denoted as

$$
A \frac{\partial U}{\partial z}+B \frac{\partial U}{\partial r}+\frac{1}{r} H=0,
$$


where $U={ }^{t}(u, v, p)={ }^{t}\left(u_{z}, u_{r}, p\right)$, and $a=(\partial p / \partial \rho)^{1 / 2} . A, B$ are corresponding matrices in (2.8), and $H=K_{0} U$ with

$$
K_{0}=\left(\begin{array}{ccc}
0 & 0 & 0 \\
0 & 0 & 0 \\
0 & 1 & 0
\end{array}\right) .
$$

To solve the system (2.10) with boundary conditions (2.4), (2.6), we introduce new coordinates

$$
x=z, \quad \alpha=\frac{r}{z}
$$

which blows up at the vertex of the cone. Obviously,

$$
\partial_{z}=\partial_{x}-\frac{\alpha}{x} \partial_{\alpha}, \quad \partial_{r}=\frac{1}{z} \partial_{\alpha}=\frac{1}{x} \partial_{\alpha} .
$$

Then in the $(x, \alpha)$ coordinates, the system (2.10) becomes

$$
A\left(\partial_{x}-\frac{\alpha}{x} \partial_{\alpha}\right) U+B \frac{1}{x} \partial_{\alpha} U+\frac{1}{r} K_{0} U=0,
$$

or

$$
(B-\alpha A) \partial_{\alpha} U+x A \partial_{x} U+\frac{1}{\alpha} K_{0} U=0 .
$$

The boundary conditions (2.4) and (2.6) will be given on $\alpha=b(x) / x$ and $\alpha=s(x) / x$.

3. Approximate solution. As the preparation of solving (2.1), (2.4) and (2.6) we use the form (2.12) to derive an approximate solution with error $O\left(x^{N}\right)$. Assume that $U(x, \alpha)$, $b(x)$ and $\sigma(x)$ have the following finite expansions

$$
U=\sum_{n=0}^{N} x^{n} U_{n}(\alpha), \quad b=\sum_{n=1}^{N+1} x^{n} b_{n}, \quad \sigma=\sum_{n=1}^{N+1} x^{n} \sigma_{n} .
$$

For the convenience of our later computations we first give the following lemma.

Lemma 3.1. Assume that $f(x)=\sum_{n=0}^{N} x^{n} f_{n}$ and $g(x)=\sum_{n=0}^{N} x^{n} g_{n}$. Then

$$
\begin{gathered}
f(x) g(x)=f_{0} g_{0}+\sum_{n=1}^{N}\left(f_{0} g_{n}+f_{n} g_{0}+P_{n}(f, g)\right) x^{n}+O\left(x^{N+1}\right), \\
F(f(x))=F\left(f_{0}\right)+\sum_{n=1}^{N}\left(F^{\prime}\left(f_{0}\right) f_{n}+P_{n}(f)\right) x^{n}+O\left(x^{N+1}\right),
\end{gathered}
$$

where $P_{n}(f, g)$ and $P_{n}(f)$ are polynomials of all $f_{i}$ and $g_{i}$ with $i<n$.

The proof of the lemma is straightforward. 
Substituting (3.1) into (2.12) and to the boundary conditions (2.4), (2.6), and then letting the coefficients of $x^{k}$ vanish for all $k<N$, we can determine all coefficients $U_{n}(\alpha), b_{n}$ and $\sigma_{n}$. From the equation (2.12) we have

$$
\begin{gathered}
(B-\alpha A)_{0} \partial_{\alpha} U_{0}+\frac{1}{\alpha} K_{0} U_{0}=0, \\
\ldots \\
(B-\alpha A)_{0} \partial_{\alpha} U_{n}+(B-\alpha A)_{n} \partial_{\alpha} U_{0}+\frac{1}{\alpha} K_{0} U_{n}+n A_{0} U_{n}=F_{n}(U),
\end{gathered}
$$

where $(B-\alpha A)_{n}$ means $(B-\alpha A)^{\prime}\left(U_{0}\right) \cdot U_{n}, F_{n}(U)$ is an expression depending on $U_{i}$ with $i<n$, and vanishes if $U_{1}=\cdots=U_{n-1}=0$.

To describe the boundary conditions of $U_{n}(\alpha)$ we use the notation

$$
\underline{U}_{n}=U_{n}\left(b_{1}\right), \quad \bar{U}_{n}=U_{n}\left(s_{1}\right) .
$$

Then the condition (2.4) is

$$
\sum_{n=0}^{N} x^{n} v_{n}\left(\frac{b(x)}{x}\right)-\sum_{n=0}^{N} b^{\prime}(x) x^{n} u_{n}\left(\frac{b(x)}{x}\right)=0,
$$

which leads to

$$
\begin{gathered}
v_{0}\left(b_{1}\right)-b_{1} u_{0}\left(b_{1}\right)=0, \\
\ldots \ldots \\
v_{n}\left(b_{1}\right)-b_{1} u_{n}\left(b_{1}\right)=g_{n}(b, \underline{u}, \underline{v}),
\end{gathered}
$$

where $g_{n}(b, \underline{u}, \underline{v})$ is an expression depending on $b_{j}(j<n+1), u_{i}, v_{i}(i<n)$, and is known in the step of determining $U_{n}(\alpha)$.

REMARK 3.1. Due to the properties of $F_{n}(U)$ and $g_{n}(b, \underline{u}, \underline{v})$ we have the fact that the assumption $b_{j}=0\left(2 \leq j \leq k_{0}\right)$ implies $U_{j}=0\left(1 \leq j \leq k_{0}-1\right)$ and $\sigma_{j}=0\left(2 \leq j \leq k_{0}\right)$.

Turning to the R-H condition on $\alpha=\sigma(x) / x$, the first three equations of (2.6) are

$$
\begin{aligned}
\rho\left(v-\sigma^{\prime} u\right) & =-\rho_{\infty} \sigma^{\prime} q_{\infty}, \\
\left(1+\sigma^{\prime 2}\right) p+\rho\left(v-\sigma^{\prime} u\right)^{2} & =\left(1+\sigma^{\prime 2}\right) p_{\infty}+\rho_{\infty} \sigma^{\prime 2} q_{\infty}^{2}, \\
u+\sigma^{\prime} v & =q_{\infty},
\end{aligned}
$$

where $q_{\infty}, \rho_{\infty}, p_{\infty}$ are the velocity, the density and the pressure of the upstream flow. Therefore, we have

$$
\begin{aligned}
\bar{\rho}_{0}\left(\bar{v}-\sigma_{1} \bar{u}_{0}\right) & =-\rho_{\infty} \sigma_{1} q_{\infty}, \\
\left(1+\sigma_{1}^{2}\right) \bar{p}_{0}+\bar{\rho}_{0}\left(\bar{v}_{0}-\sigma_{1} \bar{u}_{0}\right)^{2} & =\left(1+\sigma_{1}^{2}\right) p_{\infty}+\rho_{\infty} \sigma_{1}^{2} q_{\infty}^{2}, \\
\bar{u}_{0}+\sigma_{1} \bar{v}_{0} & =q_{\infty} .
\end{aligned}
$$

To derive the condition for $U_{n}$ we write the first three equations of (2.6) as

$$
-\sigma^{\prime}(z)[F]+[G]=0 .
$$


Then for general $n$ we have

$$
-F^{\prime}\left(U_{0}\right) U_{n} \cdot \sigma_{1}+G^{\prime}\left(U_{0}\right) U_{n}-[F] \cdot(n+1) \sigma_{n+1}=g_{n}(\sigma, U),
$$

where $g_{n}(\sigma, U)$ is an expression depending on $\sigma_{j}(j<n+1)$ and $U_{i}(i<n)$.

LEMMA 3.2. The problem (3.4), (3.6) and (3.9) correspond to the problem of the same supersonic flow past a symmetricall circle cone with vertex angle $\arctan b_{1}$. Hence, when $b_{1}$ is less than a critical value, the solution of the system (3.4) satisfying (3.6) and (3.9) exists.

ProOF. Indeed, the system (3.4) is

$$
\begin{aligned}
& \left(\begin{array}{ccc}
\rho\left(v_{0}-\alpha u_{0}\right) & & -\alpha \\
-\alpha & \rho\left(v_{0}-\alpha u_{0}\right) & 1 \\
-\alpha & 1 & a^{-2} \rho^{-1}\left(v_{0}-\alpha u_{0}\right)
\end{array}\right) \frac{\partial}{\partial \alpha}\left(\begin{array}{c}
u_{0} \\
v_{0} \\
p_{0}
\end{array}\right) \\
& +\frac{1}{\alpha}\left(\begin{array}{c}
0 \\
0 \\
v_{0}
\end{array}\right)=0 .
\end{aligned}
$$

The last equation indicates $s_{0}=$ const. Omitting subscript " 0 ", we obtain

$$
\begin{gathered}
\rho(v-\alpha u) \frac{\partial u}{\partial \alpha}-\alpha \frac{\partial p}{\partial \alpha}=0, \\
\rho(v-\alpha u) \frac{\partial v}{\partial \alpha}+\frac{\partial p}{\partial \alpha}=0, \\
-\alpha \frac{\partial u}{\partial \alpha}+\frac{\partial v}{\partial \alpha}+a^{-2} \rho^{-1}(v-\alpha u) \frac{\partial p}{\partial \alpha}+\frac{1}{\alpha} v=0 .
\end{gathered}
$$

Eliminating $\partial p / \partial \alpha$, we obtain

$$
\begin{gathered}
\alpha \frac{\partial v}{\partial \alpha}+\frac{\partial u}{\partial \alpha}=0, \\
\alpha^{2}\left(1-\frac{u^{2}}{a^{2}}\right) u_{\alpha}-\frac{2 u v \alpha^{2} v_{\alpha}}{a^{2}}-\alpha\left(1-\frac{v^{2}}{a^{2}}\right) v_{\alpha}+v=0,
\end{gathered}
$$

which is exactly the same as the system for irrotational conical flow derived in [5]. Hence the conclusion of the lemma is obtained from the Chapter VI of [5].

LEMMA 3.3. There is an integer $k_{0}$, such that the system (3.5) is a symmetric positive system in the interval $b_{1}<\alpha<\sigma_{1}$ for $n>k_{0}$. Moreover, the boundary conditions are admissible in Friedrichs' sense (see [6]). Therefore, the problem (3.5), (3.7), (3.10) admits a unique solution for $n>k_{0}$

Proof. Obviously, the coefficients matrix $(B-\alpha A)_{0}$ of $\partial_{\alpha} U_{n}$ in (3.3) is symmetric. Meanwhile, the matrix $A$ is definitely positive, if $u>a$. Therefore, (3.3) is symmetric positive system when $n$ is greater than an integer $k_{0}$, which depends only on the parameters of the coming flow and the function $b(z)$. 
Denote by $\beta$ the normal matrix $(B-\alpha A)_{0} \cdot \cos (\nu, \vec{r})$ on boundaries with $v$ being the outward normal direction to the boundary, and denote by $\pi$ the subspace of $R^{3}$ defined by the homogeneous equality corresponding to the boundary condition (3.10). Then the abmissibility on the boundary conditions in Friedrich's sense requires

$$
{ }^{t} U_{n} \beta U_{n} \geq 0,
$$

for all $U_{n} \in \pi$. Moreover, it requires that there is no other subspace $\pi_{1}$ of $R^{3}$, so that (3.14) holds on $\pi_{1}$, and $\pi$ is a proper subspace of $\pi_{1}$. Under such a condition the boundary value problem (3.5), (3.7), (3.10) is uniquely solvable. Hence what we need to do is to check the requirement (3.14) on $\alpha=b_{1}$ and $\alpha=\sigma_{1}$ respectively.

Since $\beta=-(B-\alpha A)_{0}$ and $v-\alpha u=0$ on $\alpha=b_{1}$,

$$
\beta=\left(\begin{array}{ccc}
0 & & \alpha \\
& 0 & -1 \\
\alpha & -1 & 0
\end{array}\right) .
$$

It turns out ${ }^{t} U_{n} \beta U_{n}=2\left(v_{n}-\alpha u_{n}\right) p_{n}=0$ on $\alpha=b_{1}$.

Observe the condition on the shock front $\alpha=\sigma_{1}$. $\beta=\left(B-\sigma_{1} A\right)_{0}$ on $\alpha=\sigma_{1}$. Denote

$$
\tilde{\beta}=G_{U}^{\prime}-\sigma_{1} F_{U}^{\prime}=\left(\begin{array}{ccc}
\rho w-\sigma_{1} \rho u & \rho u & a^{-2} u w-\sigma_{1} \\
-\sigma_{1} \rho v & \rho v+\rho w & 1+a^{-2} v w \\
-\sigma_{1} \rho & \rho & a^{-2} w
\end{array}\right),
$$

where $w=v-\sigma_{1} u$, the homogeneous condition corresponding to (3.10) is

$$
\tilde{\beta} U_{n}-[F] \cdot(n+1) \sigma_{n+1}=0 .
$$

Through a suitable elementary transformation of the matrix the equation (3.16) can be reduced to

$$
\beta U_{n}=(n+1) \sigma_{n+1} E,
$$

where

$$
\beta=\left(\begin{array}{ccc}
\rho w & & -\sigma_{1} \\
& \rho w & 1 \\
-\sigma_{1} & 1 & a^{-2} \rho^{-1} w
\end{array}\right), \quad E={ }^{t}(p, 0, u) .
$$

(3.17) also implies

$$
U_{n}=(n+1) \sigma_{n+1} \beta^{-1} E .
$$

Hence

$$
\begin{aligned}
{ }^{t} U_{n} \beta U_{n} & =(n+1)^{2} \sigma_{n+1}^{2}{ }^{t} E \beta^{-1} E \\
& =\frac{(n+1)^{2} \sigma_{n+1}^{2}}{\Delta}\left(\left(\frac{w^{2}}{a^{2}}-1\right) p^{2}+2 \sigma_{1} \rho w p u+\rho^{2} w^{2} u^{2}\right),
\end{aligned}
$$

where

$$
\Delta=\operatorname{det} \beta=\rho w\left(\frac{w^{2}}{a^{2}}-1-\sigma_{1}^{2}\right) .
$$


It is easy to check that $w<0$ and $|w|\left(1+\sigma_{1}^{2}\right)^{-1 / 2}$ equals $\left|u_{n}\right|$ - the normal component of velocity, which is less than the sonic speed. Hence $\Delta>0$.

Notice that $\sigma_{1}=\tan \beta$, where $\beta$ is the vertex angle of the conical shock front for the background solution. Then the quadratic form in the right side of (3.18) is

$$
\begin{aligned}
& \left(\rho w u-\sigma_{1} p\right)^{2}+\left(\frac{w^{2}}{a^{2}}-1-\sigma_{1}^{2}\right) p^{2} \\
& \quad=\sec ^{2} \beta\left(\left(\rho u\left|u_{n}\right|-p \sin \beta\right)^{2}+\left(\frac{u_{n}^{2}}{a^{2}}-1\right) p^{2}\right) .
\end{aligned}
$$

Denoting $u_{n}^{2} / a^{2}$ by $k$ and the angle of velocity with the axis by $\theta$, we have

$$
u=q \cos \theta>(\sin \beta)^{-1} q \sin (\beta-\theta)=\left|u_{n}\right| / \sin \beta .
$$

Then

$$
\begin{gathered}
\left(\rho u u_{n}-p \sin \beta\right)^{2}+ \\
\left.+\frac{u_{n}^{2}}{a^{2}}-1\right) p^{2}>\left(\frac{\gamma p u_{n}^{2}}{a^{2} \sin \beta}-p \sin \beta\right)^{2}+\left(\frac{u_{n}^{2}}{a^{2}}-1\right) p^{2} \\
=\left(\left(\frac{\gamma k}{\sin \beta}-\sin \beta\right)^{2}-(k-1)\right) p^{2},
\end{gathered}
$$

which is positive when $k=1$, and so is it when $k$ is near to 1 . It turns out that (3.19) is definitely positive in the case of weak shock. Hence ${ }^{t} U_{n} \beta U_{n} \geq 0$.

Finally, noticing that $w<0$ on $\alpha=\sigma_{1}$, the matrix $\beta$ has two negative and one positive eigenvalues. This means that any subspace $\pi_{1}$ with dimension 2 can not be a nonb-negative subspace of $\beta$. Then the unique solvability of the problem (3.5), (3.7) and (3.10) follows from [6]. Hence the proof of the lemma is complete.

According to Remark 3.1, for a given $\mathrm{b}(\mathrm{z})$ satisfying the assumption $b_{j}=0\left(2 \leq j \leq k_{0}\right)$, we have $U_{j}=0\left(1 \leq j \leq k_{0}-1\right)$ and $\sigma_{j}=0\left(2 \leq j \leq k_{0}\right)$. Since Lemma 3.3 implies that $U_{j}(j \geq k)$ can be determined by solving the boundary value problem (3.5), (3.7) and (3.10), we are led to the followoing conclusion

LEMMA 3.4. Under the assumption of Theorem 2.1, we can determine an approximate solution of (2.1), (2.4) and (2.6) with error $O\left(z^{N}\right)$ for any given number $N$.

4. Linearization. Starting from an approximate solution of the problem (2.1), (2.4) and (2.6) with error $O\left(z^{N}\right)$ obtained in the last section, we are going to use the iteration to improve the degree of the approximation and finally derive the precise solution by a limiting process.

First we make the following coordinates transformation $T$

$$
T: x=z, \quad \theta=\frac{r-b(z)}{\sigma(z)-b(z)} .
$$


The transformation fixes the free boundary $r=\sigma(z)$ to $\theta=1$, while it maps the surface $r=b(z)$ to $\theta=0$. The chain rule implies that

$$
\frac{\partial}{\partial r}=\frac{1}{\sigma-b} \frac{\partial}{\partial \theta}, \quad \frac{\partial}{\partial z}=\frac{\partial}{\partial x}+d \frac{\partial}{\partial \theta},
$$

where $d=\left\{-(\sigma-b) b^{\prime}-(r-b)\left(\sigma^{\prime}-b^{\prime}\right)\right\} /(\sigma-b)^{2}=-\left\{b^{\prime}+\theta\left(\sigma^{\prime}-b^{\prime}\right)\right\} /(\sigma-b)$. Then the problem (2.1), (2.4) and (2.6) can be rewritten as

$$
\begin{gathered}
A \frac{\partial U}{\partial x}+\left(\frac{B}{\sigma-b}+d A\right) \frac{\partial U}{\partial \theta}+\frac{1}{r} K_{0} U=0, \\
v-b^{\prime}(x) u=0 \quad \text { on } \theta=0, \\
\sigma^{\prime}(x)\left[\begin{array}{c}
\rho u \\
p+\rho u^{2} \\
\rho u v
\end{array}\right]-\left[\begin{array}{c}
\rho v \\
\rho u v \\
p+\rho v^{2}
\end{array}\right]=0 \text { on } \theta=1 .
\end{gathered}
$$

Here we have employed Bernoulli's relation to replace the last equation in (2.1) as well as the last condition in (2.6). Besides, in view of $\sigma(0)=b(0)=0$, the coefficients of $\partial / \partial \theta$ in (4.2) is singular at $x=0$. To avoid singular coefficients we also often use the form

$$
(\sigma-b) A \frac{\partial U}{\partial x}+\left(B-\left(b^{\prime}+\theta\left(\sigma^{\prime}-b^{\prime}\right) A\right) \frac{\partial U}{\partial \theta}+\frac{\sigma-b}{r} K_{0} U=0\right.
$$

instead of (4.2).

In what follows we are going to prove the existence of a solution to (4.2)-(4.4) and establish its energy estimate in an appropriate Sobolev space. Since the system (4.2) is degenerate at $x=0$, the weighted Sobolev norm with the weight $x^{-k}$ is available. To simplifying the notation we introduce a further coordinate transformation $e^{t}=x$, which transforms $x=0$ to $t=-\infty$, and $x \partial_{x}$ to $\partial_{t}$.

Write $b(x)=x \tilde{h}(x)=e^{t} h(t)$ and $\sigma(x)=x \tilde{\mu}(x)=e^{t} \mu(t)$. In the new coordinates the boundary value problem (4.2)-(4.4) becomes

$$
\begin{gathered}
\mathcal{L}(U, \mu) \equiv(\mu-h) A \frac{\partial U}{\partial t}+\mathcal{B} \frac{\partial U}{\partial \theta}+\frac{\mu-h}{h+\theta(\mu-h)} K_{0} U=0 \\
v-\left(h+h_{t}\right) u=0 \quad \text { on } \quad \theta=0 \\
\mathcal{M}_{s}(U, \mu) \equiv\left(\mu+\mu_{t}\right)[F]-[G]=0 \quad \text { on } \quad \theta=1
\end{gathered}
$$

where $\mathcal{B}=B-\left(h_{t}+\theta\left(\mu_{t}-h_{t}\right)\right) A-(h+\theta(\mu-h)) A, F$ and $G$ are the corresponding vectors obtained from (4.4).

Next, we are going to solve the problem (4.6)-(4.8) in a weighted Sobolev space. The Sobolev norms, which will be frequently employed in the sequel, are

$$
\|f\|_{k, \eta, T}^{2} \equiv \sum_{j+i_{1}+i_{2} \leq k} \int_{-\infty}^{T} \int_{0}^{1} e^{-2 \eta t} \eta^{2 j}\left|\frac{\partial^{i_{1}+i_{2}} f}{\partial^{i_{1}} t \partial^{i_{2}} \theta}\right|^{2} d t d \theta,
$$




$$
\langle f\rangle_{k, \eta, T}^{2} \equiv \sum_{j+i \leq k} \int_{-\infty}^{T} e^{-2 \eta t} \eta^{2 j}\left|\frac{\partial^{i} f}{\partial^{i} t}\right|^{2} d t .
$$

The unknowns in the problem (4.6)-(4.8) are $U(t, \theta)$ and $\mu(t)$. In order to solve the nonlinear problem we make its linearization:

$$
L(U, \mu)(\dot{U}, \dot{\mu}) \equiv(\mu-h) A \frac{\partial \dot{U}}{\partial t}+\mathcal{B} \frac{\partial \dot{U}}{\partial \theta}+E_{0} \dot{U}+E_{1} \dot{\mu}+E_{2} \dot{\mu}_{t}=\dot{f},
$$

where $E_{0}=\mathcal{L}_{U}^{\prime}, E_{1}=\mathcal{L}_{\mu}^{\prime}$ and $E_{2}=\mathcal{L}_{\mu_{t}}^{\prime}$. Besides, the linearization of the boundary conditions are

$$
\begin{gathered}
\dot{v}-\left(h+h_{t}\right) \dot{u}=0 \quad \text { on } \quad \theta=0, \\
M_{S}(U, \mu)(\dot{U}, \dot{\mu}) \equiv\left(G_{U}^{\prime}-\left(\mu+\mu_{t}\right) F_{U}^{\prime}\right) \dot{U}-[F]\left(\dot{\mu}+\dot{\mu}_{t}\right)=\dot{g} \quad \text { on } \quad \theta=1 .
\end{gathered}
$$

LEMMA 4.1. Let $k$ be any positive integer and $T<\infty$. Then there exists an $\eta_{0}>0$ such that for $\eta>\eta_{0}$, the solution $(\dot{U}, \dot{\mu})$ of the linearized problem (4.11)-(4.13) satisfies the following energy estimate

$$
\eta\|\dot{U}\|_{k, \eta, T}^{2}+\langle\dot{U}\rangle_{k, \eta, T}^{2}+\langle\dot{\mu}\rangle_{k+1, \eta, T}^{2} \leq C_{k}\left(\frac{1}{\eta}\|\dot{f}\|_{k, \eta, T}^{2}+\langle\dot{g}\rangle_{k, \eta, T}^{2}\right) .
$$

In addition, for $k \geq 2$, the constant $C_{k}$ in (4.14) depends only on the $\|\cdot\|_{k, \eta, T}^{2}$ norms of the coefficients of the equation (4.11).

Proof. Multiply (4.11) by the factor $e^{-\eta t}$ and then take the inner product of (4.11) with $e^{-\eta t} \dot{U}$ over the domain $[0,1] \times(-\infty, T)$. In view of $(\mu-h)>0$ and the definite positivity of $A$ we have

$$
\begin{aligned}
& \eta\|\dot{U}\|_{0, \eta, T}^{2}+\left\langle e^{-\eta t} \dot{U}, \mathcal{B} e^{-\eta t} \dot{U}\right\rangle_{\theta=1}-\left\langle e^{-\eta t} \dot{U}, \mathcal{B} e^{-\eta t} \dot{U}\right\rangle_{\theta=0} \\
& \quad \leq \frac{C_{01}}{\eta}\left(\|\dot{f}\|_{0, \eta, T}^{2}+\langle\dot{\mu}\rangle_{1, \eta, T}^{2}\right),
\end{aligned}
$$

where $\left.\mathcal{B}\right|_{\theta=1}=B-\left(\mu+\mu_{t}\right) A$ and $\left.\mathcal{B}\right|_{\theta=0}=B-\left(h+h_{t}\right) A$.

Like the analysis in the proof of Lemma 3.3, we estimate the boundary terms on the left side of (4.15). Because $\left.\mathcal{B}\right|_{\theta=0}$ is equal to the matrix in (3.14) with $\alpha$ replaced by $h+h_{t}$, we have

$$
\left\langle e^{-\eta t} \dot{U}, \mathcal{B} e^{-\eta t} \dot{U}\right\rangle_{\theta=0}=2\left(-\left(h+h_{t}\right) \dot{p} \dot{u}+\dot{p} \dot{v}\right)=0,
$$

while on $\theta=1$ the condition (4.13) is equivalent to

$$
\mathcal{B} \dot{U}=E\left(\dot{\mu}+\dot{\mu}_{t}\right)+\tilde{\dot{g}},
$$

where $E={ }^{t}([p], 0,[\rho u] / \rho)$, and $\tilde{\dot{g}}$ is a vector obtained from $\dot{g}$ by a linear transformation. It turns out that

$$
\begin{aligned}
& \left\langle e^{-\eta t} \dot{U}, \mathcal{B} e^{-\eta t} \dot{U}\right\rangle_{\theta=1} \\
& \quad=\left.\int_{-\infty}^{T}\left(E, \mathcal{B}^{-1} E\right) e^{-2 \eta t}\left(\dot{\mu}+\dot{\mu}_{t}\right)^{2}\right|_{\theta=1} d t-C_{02}\langle\dot{g}\rangle_{0, \eta, T}^{2} \\
& \quad=\delta\langle\dot{\mu}\rangle_{1, \eta, T}^{2}-C_{02}\langle\dot{g}\rangle_{0, \eta, T}^{2} .
\end{aligned}
$$


Then, substituting (4.17) into (4.15) and taking $\eta$ sufficiently large, we obtain (4.14) with $k=0$.

To obtain the estimate of derivatives, we first consider the case $k=1$. Denote by $D$ any tangential differential operator to the boundary $\theta=0,1$. Aplying the operator $D$ on the problem (4.11)-(4.13), we derive the estimate for $D \dot{U}$ and $D \dot{\mu}$.

The estimates of the derivatives in the normal direction can be derived from the estimates of the derivatives in the tangential direction. Since the boundary $\theta=1$ is non-characteristics, the matrix $\mathcal{B}$ is non-degenerate near $\theta=1$. Hence the estimate of derivatives in normal direction near $\theta=1$ can be obtained directly from the estimate of tangential derivatives by using (4.11). On the other hand, the matrix $\mathcal{B}$ on the boundary $\theta=0$ takes the form

$$
\left(\begin{array}{ccc}
0 & & -\left(h_{t}+h\right) \\
& 0 & 1 \\
-\left(h_{t}+h\right) & 1 & 0
\end{array}\right) .
$$

Then the estimate of

$$
\frac{\partial \dot{p}}{\partial \theta}, \quad \frac{\partial}{\partial \theta}\left(\dot{v}-\left(h+h_{t}\right) \dot{u}\right)
$$

can be derived from (4.11). Moreover, differentiating (4.11) with respect to $\theta$, we have

$$
(\mu-h) A \frac{\partial}{\partial t}\left(\frac{\partial \dot{U}}{\partial \theta}\right)+\mathcal{B} \frac{\partial}{\partial \theta}\left(\frac{\partial \dot{U}}{\partial \theta}\right)+\left(\frac{\partial \mathcal{B}}{\partial \theta}+E_{0}\right)\left(\frac{\partial \dot{U}}{\partial \theta}\right)=M,
$$

where $M$ represents a linear combination of $\dot{U}, D \dot{U}, \dot{\mu}, D \dot{\mu}$, and may have different expression in different equalities. Multiplying (4.19) by the vector $\ell=\left(1, h+h_{t}, 0\right)$, and noticing that $\ell \mathcal{B}=0$ on $\theta=0$, we obtain on the boundary $\theta=0$

$$
\rho u \frac{\partial}{\partial t}\left(\frac{\partial \dot{u}}{\partial \theta}\right)+\left(h+h_{t}\right) \rho u \frac{\partial}{\partial t}\left(\frac{\partial \dot{v}}{\partial \theta}\right)+\frac{\partial}{\partial t}\left(\frac{\partial \dot{p}}{\partial \theta}\right)=M,
$$

which yields the boundedness of the following quantity

$$
\rho u \frac{\partial \dot{u}}{\partial \theta}+\left(h+h_{t}\right) \rho u \frac{\partial \dot{v}}{\partial \theta}+\frac{\partial \dot{p}}{\partial \theta}=M .
$$

The estimates for (4.19) and (4.22) yield the estimates for all components of $\partial \dot{U} / \partial \theta$, because the determinant

$$
\left|\begin{array}{ccc}
0 & 0 & 1 \\
-\left(h+h_{t}\right) & 1 & 0 \\
\rho u & \left(h_{t}+h\right) & 1
\end{array}\right|
$$

does not vanish. Therefore, (4.14) is valid for $k=1$.

The estimates for the direvatives of higher order can be established in a similar way.

Finally, the dependency of the constant $C_{k}$ comes readily from the Banach algebra property for the Sobolev spaces $H^{k}$ with $k>[n / 2]$ and the Gagliardo-Nirenberg inequality. Hence this concludes the proof of the lemma. 
5. Local existence. In this section, we will use Newton's iteration to prove Theorem 2.1. Obviously, the existence of a curved conic shock solution in Theorem 2.1 is equivalent to the existence of a solution for boundary value problem (4.6)-(4.8). In addition, under the transformation $x=e^{t}$, a function $f(x)=O\left(x^{k}\right)$ near $x=0$ becomes $f\left(e^{t}\right)=O\left(e^{k t}\right)$ near $t=-\infty$. Therefore, denoting by $\left(U^{(0)}, \mu^{(0)}\right)\left(\mu^{(0)}=e^{-t} \sigma^{(0)}\left(e^{t}\right)\right)$ the approximate solution constructed in Section 3, we are looking for the precise solution of (4.6)-(4.8). Since the approximate solution satisfies (2.1), (2.4), (2.6) with error $O\left(x^{N}\right),\left(U^{(0)}, \mu^{(0)}\right)$ satisfies (4.6)-(4.8) with error $O\left(e^{N t}\right)$ in the $(t, \theta)$-coordinates. Here $N$ can be arbitrarily large, so the requirement of large $\eta$ in the sequel is allowed. Moreover, we may assume that (4.7) is satisfied by $U^{(0)}$ exactly. Otherwise, we can keep $u^{(0)}, p^{(0)}$ as obtaind in Section 3, and replace $v^{(0)}$ by $\left(h+h_{t}\right) u^{(0)}$. Since the error of such a replacement is only $v_{N}-\left(h+h_{t}\right) u_{N}=$ $O\left(e^{N t}\right)$, it does not change the property of error in the approximate expression of (4.6) and (4.8).

Starting from $\left(U^{(0)}, \mu^{(0)}\right)$, we use the iteration scheme

$$
\begin{gathered}
L\left(U^{(n)}, \mu^{(n)}\right)\left(\dot{U}^{(n+1)}, \dot{\mu}^{(n+1)}\right)=\dot{f}^{(n)}, \\
\dot{v}^{(n+1)}-\left(h+h_{t}\right) \dot{u}^{(n+1)}=0 \quad \text { on } \quad \theta=0, \\
M_{s}^{(n)}\left(\dot{U}^{(n+1)}, \dot{\mu}^{(n+1)}\right)=\dot{g}^{(n)} \quad \text { on } \quad \theta=1, \\
U^{(n+1)}=U^{(0)}+\dot{U}^{(n+1)}, \quad \mu^{(n+1)}=\mu^{(0)}+\dot{\mu}^{(n+1)},
\end{gathered}
$$

where

$$
\begin{aligned}
\dot{f}^{(n)}= & -\left(\mu^{(n)}-h\right) A^{(n)} \frac{\partial U^{(0)}}{\partial t}-\mathcal{B}^{(n)} \frac{\partial U^{(0)}}{\partial \theta} \\
& +E_{0}^{(n)} \dot{U}^{(n)}+E_{1}^{(n)} \dot{\mu}^{(n)}+E_{2}^{(n)} \dot{\mu}_{t}^{(n)}-\frac{\mu^{(n)}-h}{h+\theta\left(\mu^{(n)}-h\right)} K_{0} \dot{U}^{(n)} \\
\dot{g}^{(n)}= & -\mathcal{M}_{s}\left(U^{(n)}, \mu^{(n)}\right)+M_{s}^{(n)}\left(\dot{U}^{(n)}, \dot{\mu}^{(n)}\right) .
\end{aligned}
$$

Next, we are going to establish a sequence $\left\{U^{(n)}, \mu^{(n)}\right\}$ by (5.1)-(5.3) starting from $\left(U^{(0)}, \mu^{(0)}\right)$. To simplify the notation we denote the right side of (4.14) by $\|(\dot{U}, \dot{\mu})\|_{k, \eta, T}$. Then Theorem 2.1 will be proved if we can confirm the uniform boundedness in the high norm of $\left\|\left(\dot{U}^{(n)}, \dot{\mu}^{(n)}\right)\right\|_{k, \eta, T}$ with large $k$, and the contraction in the corresponding low norm with small $k$.

LEMMA 5.1. Assume that

$$
\left\|\left(\dot{U}^{(n)}, \dot{\mu}^{(n)}\right)\right\|_{k, \eta, T}<\varepsilon_{0}
$$

for small $\varepsilon_{0}$. Then for sufficiently large $\eta$, the problem (5.2)-(5.4) has a unique solution in $(-\infty, T)$ with $T \ll 0$. Moreover, the estimate (5.7) also hold with $n$ replaced by $n+1$. 
Proof. Since $\left(U^{(0)}, \mu^{(0)}\right)$ satisfies (4.6)-(4.8) with error $O\left(e^{N t}\right), \dot{f}^{(n)}$ can be written as a linear combination of $\dot{U}^{(n)}, \dot{\mu}^{(n)}, \dot{\mu}_{t}^{(n)}$ and a term $O\left(e^{N t}\right)$. Hence for $T \ll 0$,

$$
\left\|\dot{f}^{(n)}\right\|_{k, \eta, T}^{2}<C\left(\frac{1}{2} \varepsilon_{0}^{2}+\left\|\dot{U}^{(n)}\right\|_{k, \eta, T}^{2}+\left\langle\dot{\mu}^{(n)}\right\rangle_{k+1, \eta, T}^{2}\right)<2 \varepsilon_{0}^{2},
$$

where $C$ is independent of $n$. Moreover, we have

$$
\begin{aligned}
\dot{g}^{(n)} & =-\mathcal{M}_{S}\left(U^{(n)}, \mu^{(n)}\right)+\mathcal{M}_{s}\left(U^{(0)}, \mu^{(0)}\right)-\mathcal{M}_{s}\left(U^{(0)}, \mu^{(0)}\right)+M_{s}^{(n)}\left(\dot{U}^{(n)}, \dot{\mu}^{(n)}\right) \\
& =O\left(e^{N t}\right)+C^{\prime}\left(\left|\dot{U}^{(n)}\right|^{2}+\left|\dot{\mu}^{(n)}\right|^{2}\right)
\end{aligned}
$$

and

$$
\left\langle\dot{g}^{(n)}\right\rangle_{k, \eta, T}^{2} \leq \frac{1}{2}\left(\varepsilon_{0}^{2}+C^{\prime} \varepsilon_{0}^{4}\right),
$$

where $C^{\prime}$ is also independent of $n$. By using Lemma 4.1, the problem (5.1)-(5.3) is solvable. Furthermore,

$$
\left\|\left(\dot{U}^{(n+1)}, \dot{\mu}^{(n+1)}\right)\right\|_{k, \eta, T} \leq C_{k}\left(\frac{1}{\eta}\left\|\dot{f}^{(n)}\right\|_{k, \eta, T}+\left\langle\dot{g}^{(n)}\right\rangle_{k, \eta, T}\right) .
$$

Here $C_{k}$ with $k>2$ can also be chosen as a constant independent of $k$. Therefore, (5.7) with index $n+1$ holds, if $\eta$ is sufficiently large and $\varepsilon_{0}$ is sufficiently small.

REMARK 5.1. In view of $\dot{U}^{(0)}=0, \dot{\mu}^{(0)}=0$, we confirm (5.7) holds for every $n$ by induction. Therefore, for any given $k_{0}>2,\left\|\left(\dot{U}^{(n)}, \dot{\mu}^{(n)}\right)\right\|_{k_{0}, \eta, T}$ is uniformly bounded.

LEMmA 5.2. The sequence $\left\{\dot{U}^{(n)}, \dot{\mu}^{(n)}\right\}$ is convergent in the Sobolev norm $\|(\cdot, \cdot)\|_{k, \eta, T}$ with $k<k_{0}$, if the corresponding $k_{0}$-th norm is uniformly bounded ensured by Lemma 5.1.

ProOF. Let

$$
\left(\tilde{U}^{(n)}, \tilde{\mu}^{(n)}\right)=\left(\dot{U}^{(n+1)}-\dot{U}^{(n)}, \dot{\mu}^{(n+1)}-\dot{\mu}^{(n)}\right) .
$$

Consider the following boundary boundary value problem for $\left(\tilde{\dot{U}}^{(n)}, \tilde{\dot{\mu}}^{(n)}\right)$ :

$$
\begin{gathered}
L\left(U^{(n)}, \mu^{(n)}\right)\left(\tilde{U}^{(n)}, \tilde{\mu}^{(n)}\right)=\tilde{f}^{(n)}, \\
\tilde{v}^{(n+1)}-\left(h+h_{t}\right) \tilde{u}^{(n+1)}=0, \\
M_{s}^{(n)}\left(\tilde{U}^{(n+1)}, \tilde{\mu}^{(n+1)}\right)=\tilde{g}^{(n)},
\end{gathered}
$$

where

$$
\begin{aligned}
& \tilde{f}^{(n)}=\dot{f}^{(n)}-\dot{f}^{(n-1)}+L\left(U^{(n-1)}, \mu^{(n-1)}\right)\left(\dot{U}^{(n)}, \dot{\mu}^{(n)}\right)-L\left(U^{(n)}, \mu^{(n)}\right)\left(\dot{U}^{(n)}, \dot{\mu}^{(n)}\right), \\
& \tilde{g}^{(n)}=\dot{g}^{(n)}-\dot{g}^{(n-1)}+M_{s}^{(n-1)}\left(\dot{U}^{(n)}, \dot{\mu}^{(n)}\right)-M_{s}^{(n)}\left(\dot{U}^{(n)}, \dot{\mu}^{(n)}\right) .
\end{aligned}
$$

It is easy to verify that

$$
\begin{aligned}
& \frac{1}{\eta}\left\|\tilde{f}^{(n)}\right\|_{k-1, \eta, T}^{2}+\left\|\tilde{g}^{(n)}\right\|_{k-1, \eta, T}^{2} \\
& \quad \leq C^{\prime \prime}\left\|\left(\tilde{U}^{(n-1)}, \tilde{\mu}^{(n-1)}\right)\right\|_{k-1, \eta, T}^{2}\left\|\left(\dot{U}^{(n)}, \dot{\mu}^{(n)}\right)\right\|_{k, \eta, T}^{2} .
\end{aligned}
$$


Applying Lemma 4.1 to the problem (5.9)-(5.11), we have

$$
\left\|\left(\tilde{U}^{(n)}, \tilde{\mu}^{(n)}\right)\right\|_{k-1, \eta, T}^{2} \leq C_{k-1} C^{\prime \prime}\left\|\left(\tilde{U}^{(n-1)}, \tilde{\mu}^{(n-1)}\right)\right\|_{k-1, \eta, T}^{2} \cdot \varepsilon_{0}^{2} .
$$

Hence, if we choose $\varepsilon_{0}$ satisfying

$$
C_{k-1} C^{\prime \prime} \varepsilon_{0}^{2}<1,
$$

then the sequence $\left(\tilde{U}^{(n)}, \tilde{\mu}^{(n)}\right)$ is convergent in the $(k-1)$-th norm.

Proof OF Theorem 2.1. Let us now summarize the above arguments to derive Theorem 2.1. By using the result in Section 3, we construct an approximate solution of the problem (2.1), (2.4) and (2.6) with error $O\left(x^{N}\right)$. After a coordinate transformation we obtain the first approximate solution $\left(U^{(0)}, \mu^{(0)}\right)$ to the problem (4.6)-(4.8). Then the iteration scheme gives us a sequence $\left(\dot{U}^{(n)}, \dot{\mu}^{(n)}\right)$, which is convergent according to Lemma 5.2. Taking limit for $n \rightarrow \infty$, we obtain the precise solution of (4.6)-(4.8). Correspondingly, the solution of (4.2)-(4.4) in the coordinates $(x, \theta)$ and the solution of (2.1), (2.4) and (2.6) in the coordinates $(z, r)$ are also obtained. We notice that in this step we only obtain the local existence, because the condition $T \ll 0$ means that $z_{0}=x_{0}=e^{T}$ is sufficiently small.

Furthermore, the solution is stable with respect to the data, consisting of the parameters of the coming flow and $b_{1}, b_{k_{0}+1}, \ldots, b_{N+1}$. To see this we review the process of determining the solution. First, the background solution is continuously depending on the data $\rho_{\infty}, p_{\infty}, u_{\infty}$ and $b_{1}$. Since all linear problems introduced in Section 3 are stable with respect to the terms in the right hand side, the norm $\left\|U^{(0)}, \mu^{(0)}\right\|_{k+1, \eta, T}$ of the approximate solution continuously depends on the data. Besides, in the process of Newton's iteration all corresponding coefficients $C_{k}$ in (4.14), $C_{01}$ in (4.15) and $C_{02}$ in (4.18) can be chosen to be independent of the perturbation of data. Hence, denoting by $\dot{U}_{p}, \dot{\mu}_{p}$ the perturbation of $\dot{U}, \dot{\mu}$ in the iteration process, we have

$$
\begin{aligned}
& L\left(U^{(n)}, \mu^{(n)}\right)\left(\dot{U}^{(n+1)}, \dot{\mu}^{(n+1)}\right)=\dot{f}^{(n)}, \\
& L\left(U_{p}^{(n)}, \mu_{p}^{(n)}\right)\left(\dot{U}_{p}^{(n+1)}, \dot{\mu}_{p}^{(n+1)}\right)=\dot{f}_{p}^{(n)},
\end{aligned}
$$

and the corresponding equalities from the boundary conditions. From the expression (5.5) we have

$\left\|\dot{f}^{(n)}-\dot{f}_{p}^{(n)}\right\|_{k, \eta, T} \leq C\left\|U^{(0)}-\dot{U}_{p}^{(0)}, \mu^{(0)}-\dot{\mu}_{p}^{(0)}\right\|_{k+1, \eta, T}+\alpha\left\|\dot{U}^{(n)}-\dot{U}_{p}^{(n)}, \dot{\mu}^{(n)}-\dot{\mu}_{p}^{(n)}\right\|_{k, \eta, T}$.

Then, by using the energy estimate in the weighted Sobolev space we have

$$
\begin{aligned}
& \left\|\dot{U}^{(n+1)}-\dot{U}_{p}^{(n+1)}, \dot{\mu}^{(n+1)}-\dot{\mu}_{p}^{(n+1)}\right\|_{k, \eta, T} \\
& \quad \leq C\left\|U^{(0)}-\dot{U}_{p}^{(0)}, \mu^{(0)}-\dot{\mu}_{p}^{(0)}\right\|_{k+1, \eta, T}+\alpha\left\|\dot{U}^{(n)}-\dot{U}_{p}^{(n)}, \dot{\mu}^{(n)}-\dot{\mu}_{p}^{(n)}\right\|_{k, \eta, T},
\end{aligned}
$$

Inductively using (5.14) yields

$$
\left\|\dot{U}^{(n)}-\dot{U}_{p}^{(n)}, \dot{\mu}^{(n)}-\dot{\mu}_{p}^{(n)}\right\|_{k, \eta, T} \leq \frac{C}{1-\alpha}\left\|\dot{U}^{(0)}-\dot{U}_{p}^{(0)}, \dot{\mu}^{(0)}-\dot{\mu}_{p}^{(0)}\right\|_{k+1, \eta, T},
$$

which directly leads to the stability of the limit of $\left(\dot{U}^{(n)}, \dot{\mu}^{(n)}\right)$ with respect to data. Return to the original coordinate system, we confirm that the flow field $U$ and the location of the shock expressed by $r=\sigma(z)$ are stable. 


\section{REFERENCES}

[ 1] S. AlinhaC, Existence d'ondes de rarefaction pour des systèmes quasi-linéaires hyperboliques multidimensionnels, Comm. Partial Differential Equations 14 (1989), 173-230.

[ 2 ] S. X. Chen, Existence of local solution to supersonic flow past a three-dimensional wing, Adv. in Appl. Math. 13 (1992), 273-304.

[ 3 ] S. CHEN, Non-symmetric conical supersonic flow, Hyperbolic problems: theory, numerics, applications, (Zürich, 1998), 149-158, Internat. Ser. Numer. Math. 129, Birkhauser Verlag, Basel/Switzerland, 1999.

[ 4 ] S. X. ChEn AND D. LI, Supersonic flow past a symmetrically curved cone, Indiana Univ. Math. Journal 49 (2000), 1411-1435.

[ 5 ] R. Courant AND K. O. Friedrichs, Supersonic Flow and Shock Waves, Interscience Publishers Inc., New York, 1948.

[ 6 ] K. O. Friedrichs, Symmetric positive linear differential equations, Comm. Pure Appl. Math. 11 (1958), $333-418$.

[ 7 ] C. H. GU, A method for solving the supersonic flow past a curved wedge, Fudan J. (Natur. Sci.) 7 (1962), $11-14$.

[ 8 ] P. D. LAX AND R. S. PhILlips, Local boundary conditions for dissipative symmetric linear differential operators, Comm. Pure Appl. Math. 13 (1960), 427-454.

[ 9 ] D. LI, Nonlinear initial-boundary value problem for quasilinear hyperbolic system, Chinese Ann. Math. Ser. B 7 (1986), 147-159.

[10] T. T. LI, Une remarque sur un problème à frontière libre, C. R. Acad. Sc. Paris, Ser. A 289 (1979), 99-102

[11] T. T. LI, On a free boundary problem, Chinese Ann. Math. 1 (1980), 351-358.

[12] T. T. LI AND W. C. YU, Boundary value problem for quasi-linear hyperbolic systems, Duke Univ. Math., Ser. 5, Duke University, Mathematics Department, Durham, N.C., 1985.

[13] W. C. LiEn AND T. P. LiU, Nonlinear stability of a self-similar 3-dimensional gas flow, Comm. Math. Phys. 204 (1999), 525-549.

[14] A. MAJDA, The stability of multi-dimensional shock fronts, Mem. Amer. Math. Soc. 273 (1983).

[15] A. MAJDA, The existence of multi-dimensional shock fronts, Mem. Amer. Math. Soc. 281 (1983).

[16] A. MAJDA, One perspective on open problems in multi-dimensional conservation laws, Multidimensional hyperbolic problems and computations (Minneapolis, MN 1989), 217-238, IMA Vol. Math. Appl. 29, Springer, New York, 1991.

[17] A. Majda And E. Thomann, Multi-dimensional shock fronts for second order wave equations, Comm. Partial Differential Equations 12 (1987), 777-828.

[18] M. MNIF, Probléme de Riemann pour une loi de conservation scalaire hyperbolique d'order deux, Comm. Partial Differantial Equations, 22 (1997), 1589-1627.

[19] C. S. MorawetZ, Potential theory for regular and Mach reflection of a shock at a wedge, Comm. Pure Appl. Math. 47 (1994), 593-624.

[20] A. I. RYKov, Possible regimes of flow around pointed bodies of sinite thickness for arbitrary hyperbolic velocities of the incoming flow, Journal of Appl. Math. Mech. 55 (1992), 74-78.

[21] D. G. SCHAEFFER, Supersonic flow past a nearly straight wedge, Duke Math. J. 43 (1976), 637-670.

[22] D. G. SCHAEFFER, An application of the Nash-Moser theorem to a free boundary problem, (Proc. Special Sem. Indiana Univ., Bloomington, Ind., 1976-1977), 129-143, Lecture Notes in Math. 648, Springer, Berlin, 1978.

[23] J. SMOLLER, Shock waves and reaction-diffusion equations, Springer-verlag, New York, 1983.

[24] G. B. Whitham, Linear and Nonlinear Waves, John Wiley and Son, New York, London, Sydney, Toronto, 1974.

INSTITUTE OF MATHEMATICS

FUDAN UNIVERSITY

SHANGHAI

CHINA

E-mail address: sxchen@fudan.ac.cn 